\title{
A Note on Morphological Differences between Strains of Streptococcus cremoris
}

\author{
By H. R. WHITEHEAD AND G. J. E. HUNTER \\ Dairy Research Institute, Palmerston North, New Zealand
}

SUMMARY: Some strains of Streptococcus cremoris are slightly more heat resistant than the majority. After growth in milk at $37^{\circ}$ for $5 \mathrm{hr}$. some of them show under the microscope involution forms which are sufficiently characteristic to serve as a useful means of identification.

A number of strains of Streptococcus cremoris peculiarly suited for use as 'starters' in Cheddar cheese manufacture were selected from hundreds isolated during the past 12 years. The strains were selected for:

(1) great activity in the fermentation of lactose in milk culture and in cheese curd;

(2) power to withstand an incubation temperature of $37^{\circ}$ without suffering as much damage as most strains suffer;

(3) total absence of any relation to each other in their reactions to a series of bacteriophages.

The cultures have been regularly plated and repurified at intervals of about 2 months (some of them over seventy times), so that their characters are likely to be as stable as possible. There is evidence, however, that during the period of observation the rate of acid production decreased slightly in some of them. Since all the strains belong to one species there are not many features by which they may with certainty be distinguished. There are, however, some apparently characteristic morphological differences between them which have proved exceedingly valuable, not only in distinguishing these particular strains but also in enabling us to predict whether a freshly isolated unknown strain is related to any of them.

\section{The demonstration of morphological differences}

The optimum temperature for growth of most strains of Strep. cremoris in milk is in the region of $30^{\circ}$; at $37^{\circ}$ the organisms still grow but are somewhat inhibited. Subjected to the latter rather unfavourable temperature the various strains exhibit differences in morphology which have a value in identification. The stock strains when grown in skim milk at $22^{\circ}$ or $30^{\circ}$ cannot be distinguished from one another under the microscope at any stage of growth.

Figs. 1-3, Pl. 1, illustrate the appearance of the three strains, HP, K and R 6 after incubation for $5 \mathrm{hr}$. at $30^{\circ}$. All the strains form the long chains of spherical cocci typical of Strep. cremoris. When, however, the organisms are incubated in milk culture at $37^{\circ}$ for $5 \mathrm{hr}$. they assume forms which are characteristic enough to serve as identifying features. Thi sis illustrated in Figs. 5-7 (Pl. 1), where the following points may be noted. 
Culture $\boldsymbol{H P}$. Cocci still spherical, but terminal cocci in many chains are swollen to about twice normal size (Fig. 5).

Culture $K$. Cocci have assumed flat-sided squarish shape, and the chains have a characteristic tangled arrangement (Fig. 6).

Culture $\boldsymbol{R}_{6}$. Very long chains of flattened cocci crushed together concertinafashion (Fig. 7).

All the cultures grow less at $37^{\circ}$ than at $30^{\circ}$, a reflexion of the unsuitability of $37^{\circ}$ as a growth temperature for Strep. cremoris.

The involution shapes of several other strains of Strep. cremoris are similarly quite characteristic, and on many occasions served as a means of predicting that an unknown culture was related to one of the stock strains. The three strains described can be identified with certainty by the method described. Other strains show less definite changes in morphology which, nevertheless, are capable of recognition by the experienced worker; still other active strains cannot be positively identified by this means. A large number of strains of Strep. cremoris which are not active enough to be used as cheese 'starters' are much more heat sensitive and react to incubation at $37^{\circ}$ as shown in Figs. 4 and 8 (Pl. 1). It will be observed that whereas this strain shows a healthy growth at $30^{\circ}$, it is very feeble and badly involuted at $37^{\circ}$.

Distinction between Strep. cremoris and Strep. lactis

Although many workers still do not agree that Strep. cremoris is distinct as a species from $S$ trep. lactis we find that most lactis types which ferment maltose and dextrin and which form only diplococci or very short chains in culture, also give quite a different appearance from our stock cremoris strains after growth at $37^{\circ}$. They are not significantly inhibited even at $37^{\circ}$, and the cocci (smaller even at $30^{\circ}$ than those of Strep. cremoris) are not significantly altered in shape or arrangement at the higher temperature. This is in accord with expectation, since strains of Strep. lactis have in general a higher optimum and maximum growth-temperature than strains of Strep.cremoris. Thus it is usually possible on the basis of morphology at different incubation temperatures to divide lactic streptococci into groups which correspond with Orla-Jensen's species Strep. lactis and Strep. cremoris, and these groups are also characterized respectively by ability and inability to ferment maltose and by a different pattern of sensitivity to various phages (Hunter, 1946). There is, however, a small proportion of intermediate types which cannot be definitely assigned to either species.

\section{DISCUSSION}

The characteristic morphology assumed by many strains of Strep. cremoris after incubation in milk at $37^{\circ}$ for $5 \mathrm{hr}$. is a very useful criterion, both for selecting active and heat-resistant cultures and for predicting the phage relationships between strains. In the course of a search extending over several years for unrelated strains of Strep. cremoris we have gained the impression that the number of types is relatively limited. Thus only seven unrelated strains 
Journal of General Microbiology, Vol. 3, No. 1
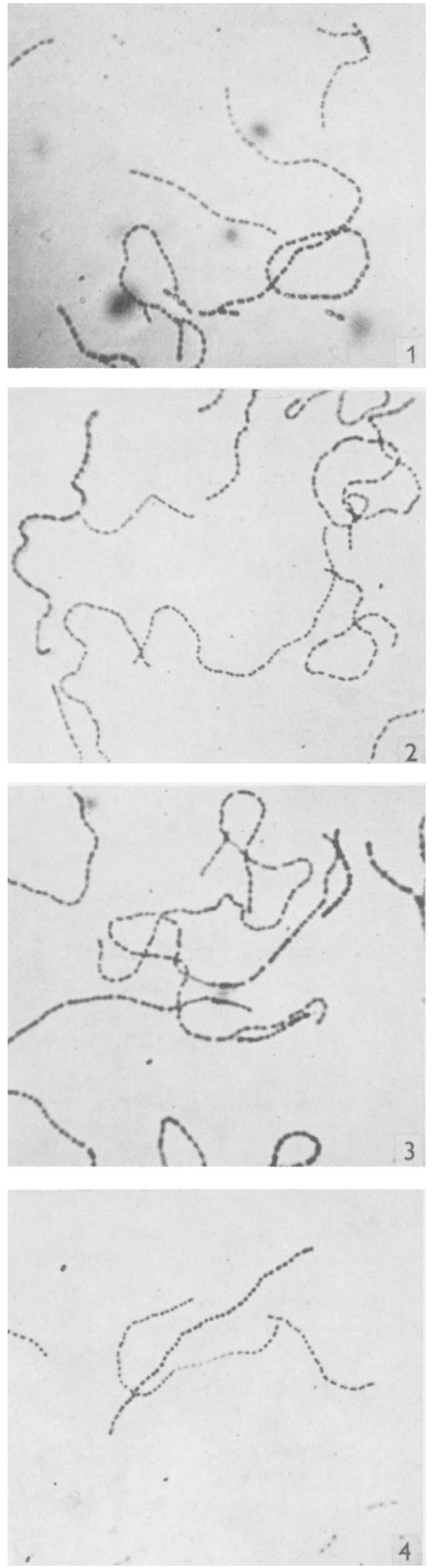
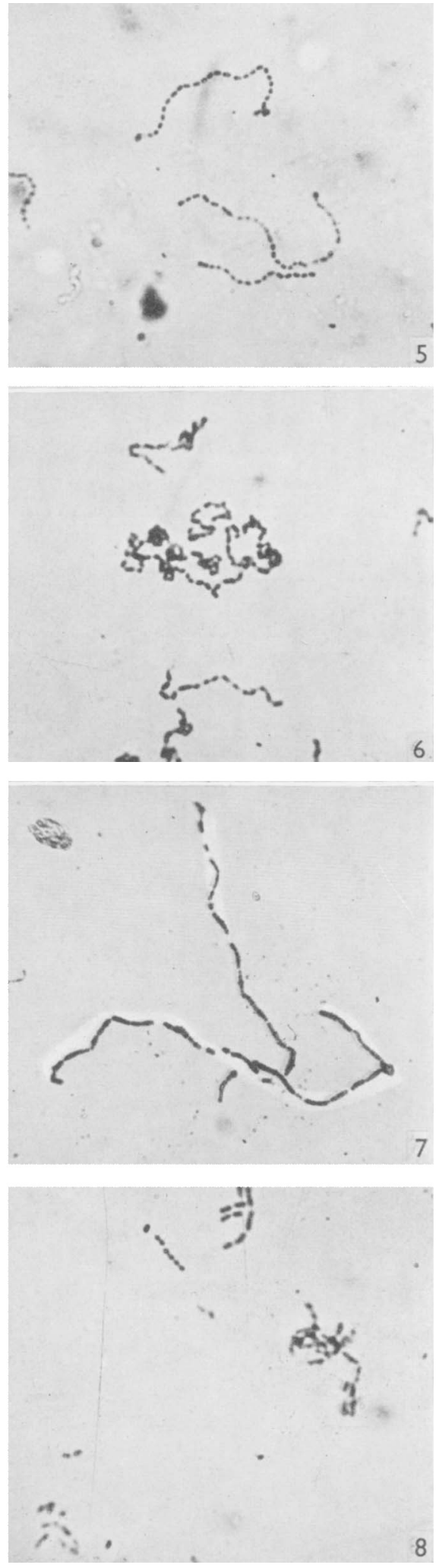

Figs. 1-8

H. R. Whithiead \& G, J. E. Hunter-Morphology of Strkp, cremorts strains, Plate I 
active enough for our purpose have been found over the course of about 10 years. It is, however, possible that within any one country a small group of types becomes widespread, with the result that the same strains are constantly being encountered. There are some indications that unrelated strains are more easily found in material from abroad. We hope to investigate this possibility further.

\section{REFERENCE}

Hunter, G. J. E. (1946). The differentiation of Streptococcus cremoris and Streptococcus lactis by means of bacteriophage action. J. Hyg., Camb., 44, 264.

\section{EXPLANATION OF PLATE}

(Magnification $\times 900)$

Figs. 1-3. Strep. cremoris cultures $H P, K$ and $R_{6}$ after 5 hr. growth at $30^{\circ}$.

Figs. 5-7. The same cultures after $5 \mathrm{hr}$. growth at $37^{\circ}$.

Figs. 4, 8. A heat-sensitive strain of Strep. cremoris after growth at 30 and $37^{\circ}$ respectively for $5 \mathrm{hr}$.

(Received 23 March 1948) 